\title{
Prediction of the Steady Rate of Flame Spread Over Combustible Materials
}

\author{
A. I. KARPOV and V. K. BULGAKOV \\ Khabarovsk State University of Technology \\ Tikhookeanskaya, 136 \\ Khabarovsk 680035 \\ Russia
}

\begin{abstract}
The propagation of the two-dimensional diffusive flame over combustible material is studied by solving appropriate steady-state conservation equations written in the coordinate system fixed on the flame front where the flame spread rate appears as an eigenvalue. It is shown that a solid fuel's mass balance is kept for any assigned value of the flame spread rate and therefore, the eigenvalue problem has not unique solution. A new algorithm for the flame spread rate prediction is proposed using the non-equilibrium thermodynamic approach. The steady flame spread is identified with the stationary thermodynamic state which is characterized, according to the theorem of Prigogine, by the minimal entropy production. The influence of free-stream velocity and ambient oxygen mass fraction on the flame spread process over thin sheets of paper is investigated.
\end{abstract}

KEYWORDS - Flame spread; Irreversible thermodynamic process; Numerical modeling;

\section{NOMENCLATURE}

$A_{s}$ - Chemical affinity of pyrolysis reaction;

a - Gas-phase reaction's order with respect to fuel;

b - Gas-phase reaction's order with respect to oxidizer;

C - Specific heat;
$E$ - Activation energy;

$J_{i}$ - Thermodynamic flux of $i$-th irreversible process;

$J_{k}$ - Diffusive flux of $\boldsymbol{k}$-th component;

$k$ - Preexponential factor;

$L$ - Solid fuel half-thickness; 
Le - Lewis number;

$P$ - Bulk entropy production;

$p$ - Pressure;

$Q$ - Effective heat of reaction;

$q$ - Heat flux;

$\boldsymbol{R}$ - Specific gas constant;

$\boldsymbol{R}_{\mathbf{0}}$ - Universal gas constant;

$\boldsymbol{S}$ - Entropy;

$T$ - Temperature;

$\boldsymbol{U}$ - Internal energy;

$\boldsymbol{u}$ - Velocity component in the $x$-coordinate;

$u_{i}$ - Velocity vector;

$u_{f}$ - Flame spread rate;

\section{Greek}

$\delta$ - Thickness of gasified solid fuel;

$\lambda$ - Thermal conductivity;

$\mu_{k}$ - Chemical potential of $\boldsymbol{k}$-th component;

\section{Subscripts}

$a$ - Ambient;

$F$ - Fuel;

$O$ - Oxidizer;

$s$ - Solid phase;
$V$ - Specific volume;

$v$ - Velocity component in the $\boldsymbol{y}$-coordinate;

$v_{n}$ - Normal buming rate (Fig.2);

$v_{s}$ - Linear pyrolysis rate;

$W$ - Chemical reaction rate;

$X_{i}$ - Generalized force of $i$-th irreversible process;

$x$ - Coordinate along the fuel's surface;

$x_{b}$ - Burn-out position;

$x_{i}$ - Coordinate vector;

$y$ - Coordinate normal to the fuel's surface;

$Y_{k}$ - Mass fraction of $k$-th component;

$v_{k}-$ Stoichoimetric coefficient of $k$-th component;

$\rho$ - Density;

$\rho_{s}^{\prime}$ - Variable density of solid fuel;

$\tau_{i j}$ - Shear stress tensor;

$T$ - Heat transfer;

$v$ - Vaporization;

$W$ - Chemical reaction;

\section{INTRODUCTION}

The knowledge of the flame spread velocity, the basic parameter characterizing the flame spread process [1-4], provides a most valuable information on the overall effect of flame propagation esspecially in the sense of practical application to fire safety. Furthermore, the flame spread velocity is the single parameter that can be measured directly, quite accurately and rather easily in comparison with the other characteristics such a flame temperature or species mass fractions. As a rule, flame spread rate values obtained by different authors are correlated well, at least for the materials with known properties. Therefore, every theoretical model faces the most important requirement: it must be able to predict the flame spread rate. Analysing the results [5-14] reported during the last decades it may be concluded that the determination of the flame spread rate, being a perfectly feasible for experiment, is the most difficult task for theory. A lot of models have been developed to reach this goal but only few of them have been carried far enough to predict the spread rate theoretically itself, i.e. without employing an empirical data which are valid for some specific experiment only. Some studies of such level have been selected for the analysis in the next section. 


\section{MODELS OF THE STEADY FLAME SPREAD}

The typical flame spread model is shown in Fig.1. The most general form of the governing conservation equations written in the coordinate system fixed on the flame front is following:

gas phase -

$$
\begin{aligned}
& \rho u_{j} \frac{\partial u_{i}}{\partial x_{j}}=\frac{\partial \tau_{i j}}{\partial x_{j}}-\frac{\partial p}{\partial x_{i}} \\
& C \rho u_{j} \frac{\partial T}{\partial x_{j}}=-\frac{\partial q_{j}}{\partial x_{j}}+Q \rho W \\
& \rho u_{j} \frac{\partial Y_{k}}{\partial x_{j}}=-\frac{\partial J_{k j}}{\partial x_{j}}-v_{k} \rho W \\
& \frac{\partial \rho u_{i}}{\partial x_{i}}=0
\end{aligned}
$$

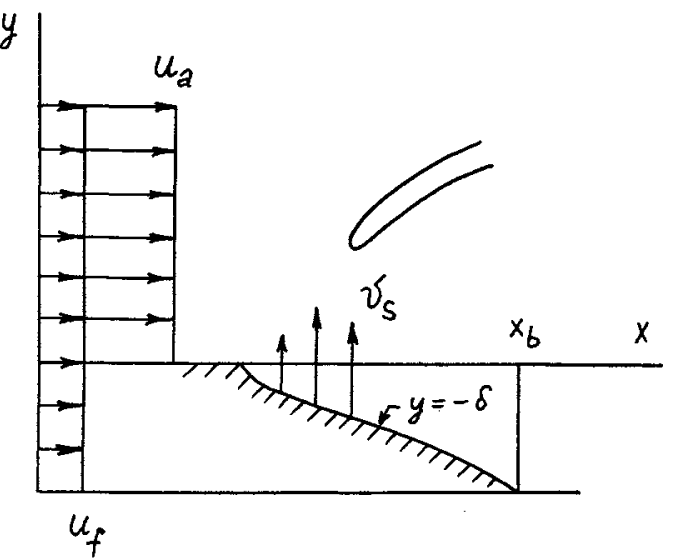

$p=\rho R T$

Fig.1 Flame spread model

solid fuel -

$$
C_{s} \rho_{s} u_{f} \frac{\partial T_{s}}{\partial x}=\lambda_{s}\left(\frac{\partial^{2} T_{s}}{\partial x^{2}}+\frac{\partial^{2} T_{s}}{\partial y^{2}}\right)+\rho_{s} Q_{s} W_{s}
$$

boundary conditions -

$y=0: \quad T=T_{s}$

$-\lambda \frac{\partial T}{\partial y}+\rho v C T=-\lambda_{s} \frac{\partial T_{s}}{\partial y}+\rho_{s} \nu_{s} C_{s} T_{s}$

$-\frac{L e \lambda}{C} \frac{\partial Y_{k}}{\partial y}+\rho v Y_{k}=\rho_{s} v_{s} Y_{k, s}$

$\rho \nu=\rho_{s} v_{s}$

$u=u_{f}$ 


$$
y=-L: \quad T_{s}=T_{s, a} \quad \text { or } \quad \partial T / \partial y=0
$$

The gas-phase combustion reaction and solid fuel pyrolysis both are assumed to be described by Arrhenius-type formulas:

$$
\begin{aligned}
& W=k Y_{F}^{a} Y_{O}^{b} \exp \left(-E / R_{0} I\right) \\
& W_{s}=k_{s} \exp \left(-E_{s} / R_{0} T_{s}\right)
\end{aligned}
$$

The mathematical statement defined by Eqs.(1)-(6) provides six equations for six variables which are $u_{i}, T, Y_{k}, p, \rho, T_{s}$. Therefore, two additional equations are necessary to obtain the other unknown parameters: pyrolysis rate $v_{s}$ and steady flame spread rate $u_{f}$. The nature of the first of these equations is obvious since the solid fuel's mass conservation is not included in the Eqs.(1)-(6) while the expression for the obtaining of the flame spread rate is the cornerstone of steady flame spread model.

There are models $[12,13]$ that keep the unsteady terms in conservation equations written in the laboratory coordinate system. In this case the eigenvalue problem is avoided and the mathematical statement of problem is specified properly. The steady flame spread rate is obtained here when the stationary movement of flame front is achieved. The undoubted advantage of such approach is obvious since the characteristics of processes that are non-stationary in nature such an ignition or other time-dependent change of parameters can be obtained. On the other hand, the modeling of steady-state process by solving the set of unsteady equations does not seem to be the most advanced meaning on the CPU time requirements. However, this opinion can not be verified or, perhaps, refuted now because the alternative object for comparison is not outlined clearly. It means that there is no any generally appointed algorithm for the prediction of steady flame spread rate in terms of steady-state equations written in the flame front fixed coordinate system.

Relatively to the eigenvalue problem arising in this case the essence of pioneering study of de Ris [5] consists in the introduction of the temperature of solid fuel vaporization $T_{y}$. In the further studies the more accurate (Wichman and Williams [9]) and exact (Delichatsios [10]) solutions of de Ris' model have been obtained. However, the boundary conditions expressed by Eqs.(7)-(8) determine the surface temperature uniquely as a result of solution of the coupled energy equations (2) and (6) and the artificial condition $T=T_{v}$ on $y=0$ can not replace either Eq.(7) or Eq.(8). The reader is addressed to the survey paper of Sirignano [7] for detailed discussion.

In his own turn, Sirignano [8] proposed the model where the concept of the vaporization temperature has been eliminated. The fuel mass balance is used here to obtain the steady flame spread rate in the following form: 


$$
\rho_{s} u_{f} L=\int_{-\infty}^{x_{b}} \rho_{s} v_{s} d x
$$

Here the finite value of $x_{b}$ indicating the bum-out point beyond which no pyrolysis reaction occurs is taken arbitrary since the solution is obtained assymptotically as $x_{b} \rightarrow \infty$. In the sense of eigenvalue problem the model of Sirignano is specified properly but limited by the assumption that there is no influence of the bum-out position on the flame spread process (actually, the typical peculiarity for the flame spread over thermally thick fuel beds). Therefore, since this assumption can not be invoked elsewhere, this model can not be appointed as a general. A quite clear evidence of this limitation can be carried out by analyzing the recent study of Bhattacharjee and Altenkirch [14] where the similar approach is used. The material's regression (described by the density loss) is not neglected here and burn-out position is introduced explicitly disregarding the remedies by which this point can be obtained in advance. The discussion $[12,15]$ on Sirignano's model notes usually the limitation due to the only surface reaction is considered. However, this fact relates to the solution method of differential equations because an analytical solution (here by Viener-Hopf technique) can not be obtained in the case of bulk reaction with finite kinetics. Concerning the problem of flame spread rate prediction the analysis of Sirignano is not limited by the reaction's nature and it, perhaps, stands mostly close to the understanding of the high complexity of the considered eigenvalue problem. It is confirmed implicitly by the fact that his initial hopeful model [7] was followed within two years by the more detailed analysis [8] where the limitations of the model are realized.

\section{EIGENVALUE PROBLEM}

Let start from the simple mass-loss formula:

$$
\frac{d \rho_{s}^{\prime}}{d t}=-\rho_{s} W_{s}
$$

Here pyrolysis reaction's rate is defined by Eq.(15). Transforming Eq.(17) to the steady-state coordinate system fixed on the flame front we have

$$
u_{f} \frac{d \rho_{s}^{\prime}}{d x}=-\rho_{s} W_{s}
$$

Integration of Eq.(18) in some cross-section on $x$-coordinate yields

$$
u_{f} \frac{d}{d x}\left(\int_{-L}^{-\delta} \rho_{s}^{\prime} d y+\int_{-\delta}^{0} \rho_{s}^{\prime} d y\right)=-\rho_{s}\left(\int_{-L}^{-\delta} W_{s} d y+\int_{-\delta}^{0} W_{s} d y\right)
$$

where position $\delta$ is identified below.

Here, the physical description of combustible material must be clarified. Since the mass 
conservation in the liquid is determined by the continuity law in which the motion is included as well as the vaporization, Eq.(19) fails if applied to the liquid fuel. Therefore, following analysis is applicable to the solid fuels only.

By the one approach, the solid combustible material is considered to be of the constant density while fuel bed thickness decreases along the $x$-coordinate due to regression of surface which position corresponds to line $y=-\delta$ in Fig.1. From the other point of view the surface regression is not considered while the nature of pyrolysis reaction is modeled by the density loss along the buming surface. Below, the first model is choosen, mainly due to its clear geometrical interpretation. Thus, since the position $y=-\delta$ denotes the interphase surface, we have $\rho_{s}^{\prime}=\rho_{s}$ for $y<-\delta$ and $\rho_{s}^{\prime}=0, W_{s}=0$ for $y>-\delta$ and Eq.(19) becomes

$$
u_{f} \frac{d \delta}{d x}=\int_{-L}^{-8} W_{s} d y
$$

The right-hand term of Eq.(20) represents the linear pyrolysis rate

$$
v_{s}=\int_{-L}^{-8} W_{s} d y
$$

where pyrolysis reaction rate $W_{s}$ is known function of temperature through Eq.(15) while temperature itself is determined by Eq.(6) with the flame spread rate $u_{f}$ included. Finally, we get the following expression for surface regression

$$
\frac{d \delta}{d x}=\frac{v_{s}}{u_{f}}
$$

Hence, now we have three equations (6),(21) and (22) for four unknown parameters $T_{s}, v_{s}, u_{f}, \delta$ and no step is made to the determination of the flame spread rate. This hopeless conclusion is not surprising because it is obvious that no more physical laws than conservation of energy and mass can be drawn up for the media like considered here. Thus, since the mass balance was used once for the determination of linear pyrolysis rate in the form of Eq.(21) there is no room for obtaining another parameter from the same physical law. Manipulation like described above allows to get another correlation, Eq.(22), with obligatory appearence of new unknown variable, here $\delta$. One of the possible ways of solution leads to the assignment of surface profile while Eq.(22) would be used for the obtaining of flame spread velocity. Such approach stands close to the assignment of vapourization (ignition) temperature, burn-out position or other artificial parameters which are not independent themselves but should be determined by self-contained solution of Eqs.(1)-(6).

The essence of Eq.(22) is of the most importance. The propagation of diffusive flame over combustible material is fundamentally two-dimensional from the view-point of coordinate system used in the model shown in Fig.1. The limitation of such approach is carried out from Eq.(22) in the clearest form. The flame, having no impression on the Cartesian (or any other) coordinate system that human uses, propagates in the direction of least resistance of 
flammability. 1 Therefore, the most attractive approach to the theoretical modeling tends to writing the conservation equations in the flame-oriented coordinates $n$ and $\theta$ as shown in Fig.2. In this case the eigenvalue problem would be transformed to the one-dimensional. Unfortunatelly, we have no any idea how it can be done and following analysis is turned into another direction.

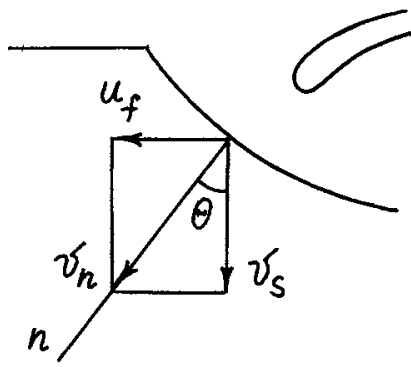

Fig.2

\section{PROPOSED APPROACH}

According to the Second Law of thermodynamics [18-20] irreversible processes drive macroscopic system to an equilibrium state at which the entropy reaches maximal value and internal entropy production becomes zero. In the case of open system when exchange of energy and matter with the surroundings is maintained in time the equilibrium state can not be achieved and system evolves to the non-equilibrium stationary state. By the approach of Prigogine [19-22] the assumption of "local equilibrium" is proposed which asserts that the Gibbs equilibrium relation

$$
T d S=d U+p d V-\sum_{k=1}^{N} \mu_{k} d Y_{k}
$$

is valid in non-equilibrium if deviation from equilibrium is small. Hence, the minimal entropy production principle is derived [19-22]

$$
P=\int_{V}\left(\sum_{i} J_{i} X_{i}\right) d V \rightarrow \min
$$

which asserts that the amount of entropy produced internally by the system is minimal in stationary state. Thus, considering the combustion as a set of irreversible processes producing entropy, the steady flame propagation is identified with the stationary state of non-equilibrium thermodynamic system characterized by the minimal entropy production. This approach was applied [23] to the prediction of the velocity of one-dimensional, premixed gas flame propagation. The observed results are quite satisfactory and further investigation is presented below for the prediction of steady rate of flame spread over thin paper sheets in a flow opposing the flame propagation. This process has been investigated in a large number of studies [24-28] and there is no any essential contradiction in experimental data on flame spread velocity obtained by different authors. The mathematical model is based on the folowing assumptions employed usually for such flame spread mode [12,29]:

\footnotetext{
1 Some studies $[16,17]$ stand close to the understanding of this peculiarity but have not carried far enough to provide a theoretical description.
} 
- material thickness is assumed be thermally thin that asserts the constant temperature distribution across the solid fuel;

- momentum equation (1) is eliminated from the calculations assuming the uniform profile for velocity component $u$ and zero value for cross-flow velocity $v$.

The validity of last assumption (instead of more realistic, on the first sight, approximation $\rho v=\rho_{s} v_{s}$ ) has been verified by Frey and Tien [29]. Hence, the governing equations are simplified to the following form:

gas phase -

$u=u_{f}+u_{a}$

$v=\mathbf{0}$

$C \rho u \frac{\partial T}{\partial x}=\lambda\left(\frac{\partial^{2} T}{\partial x^{2}}+\frac{\partial^{2} T}{\partial y^{2}}\right)+Q \rho W$

$\rho u \frac{\partial Y_{o}}{\partial x}=\frac{\operatorname{le} \lambda}{C}\left(\frac{\partial^{2} Y_{o}}{\partial x^{2}}+\frac{\partial^{2} Y_{o}}{\partial y^{2}}\right)-v_{o} \rho W$

$$
\rho u \frac{\partial Y_{F}}{\partial x}=\frac{L e \lambda}{C}\left(\frac{\partial^{2} Y_{F}}{\partial x^{2}}+\frac{\partial^{2} Y_{F}}{\partial y^{2}}\right)-v_{F} \rho W
$$

solid fuel -

$$
C_{s} \rho_{s} \mu_{f} \frac{d T}{d x}=\lambda_{s} \frac{d^{2} T}{d x^{2}}+Q_{s} \rho_{s} W_{s}
$$

The boundary conditions on the solid fuel's surface are described by Eqs.(7)-(11) where $Y_{O, s}=0, Y_{F, s}=1$. The flame spread rate is predicted by using the expression for entropy production in the solid fuel which can be expressed as follows (see Appendix):

$$
P=\int_{0}^{x_{b}} \int_{-L}^{-\delta}\left[\frac{\lambda_{s}}{T_{s}^{2}}\left(\frac{\partial T_{s}}{\partial x}\right)^{2}+\frac{Q_{s} \rho_{s} W_{s}}{T_{s}}\right] d y d x
$$

Finally, the algorithm of flame spread rate prediction is described by the following procedure. The set of Eqs.(27)-(30) has a continuum of solutions corresponding to every assigned value of spread rate $u_{f}$. Among them the unique value is searched which provides the minimal entropy production according to integral (31). 
The system of differential equations is solved by using the finite-difference scheme of Patankar [30].

\section{RESULTS AND DISCUSSION}

The gas phase and solid fuel properties listed in Table 1 have been approved $[12,29]$ for the numerical modeling of flame spread over filter paper.

TABLE 1. Gas Phase and Solid Fuel Properties [12,29]

\begin{tabular}{|c|c|c|c|c|}
\hline Name & Symbol & Gas & Solid & Unit \\
\hline Specific heat & C & 1005.6 & 1257 & $\mathrm{~J} /(\mathrm{kg} \cdot \mathrm{K})$ \\
\hline Thermal conductivity & $\lambda$ & 0.0254 & 0.1257 & $\mathrm{~W} /(\mathrm{m} \cdot \mathrm{K})$ \\
\hline Density & $\rho$ & $1.19^{a}$ & 650 & $\mathrm{~kg} / \mathrm{m}^{3}$ \\
\hline Pressure & $p$ & $10^{5}$ & - & $\mathrm{Pa}$ \\
\hline $\begin{array}{l}\text { Effective heat of } \\
\text { reaction }\end{array}$ & $Q$ & $1.68 \cdot 10^{7}$ & $-7.54 \cdot 10^{5}$ & $\mathrm{~J} / \mathrm{kg}$ \\
\hline Preexponential factor & $k$ & $3.7 \cdot 10^{7}$ & $.10^{10}$ & $1 / \mathrm{s}$ \\
\hline Activation energy & $E$ & 62850 & 125700 & $\mathrm{~J} /$ mole \\
\hline $\begin{array}{l}\text { Reaction order: } \\
\text { fuel } \\
\text { oxidizer }\end{array}$ & $\begin{array}{l}a \\
b\end{array}$ & $\begin{array}{l}1.0 \\
1.0\end{array}$ & $\begin{array}{l}- \\
-\end{array}$ & \\
\hline $\begin{array}{l}\text { Stoichoimetric } \\
\text { coefficient: } \\
\text { fuel } \\
\text { oxidizer }\end{array}$ & $v_{F}$ & $\begin{array}{l}1.0 \\
1185\end{array}$ & - & \\
\hline $\begin{array}{l}\text { oxidizer } \\
\text { Ambient temperature }\end{array}$ & $\begin{array}{l}v_{o} \\
T_{a}\end{array}$ & $\begin{array}{l}1.18 J \\
300\end{array}$ & $\begin{array}{l}- \\
-\end{array}$ & $\mathrm{K}$ \\
\hline
\end{tabular}

${ }^{a}$ at the ambient temperature

Figures 3 and 4 present the results of calculations describing the influence of some parameters on the flame spread rate. As it is shown in Fig. 3 the well-investigated dependence of flame spread rate upon the ambient oxygen mass fraction is performed well by the proposed algorithm. Such conclusion relates to the overall effect described by the whole curve including the region near flame extinction. Concerning the influence of free-stream velocity on the flame spread rate shown in Fig.4 it can be noticed that obtained results are in quite satisfactory agreement with measurements in the region of stable flame spread. The flame extinction peculiarities are not handled by present calculations in details but the critical value 


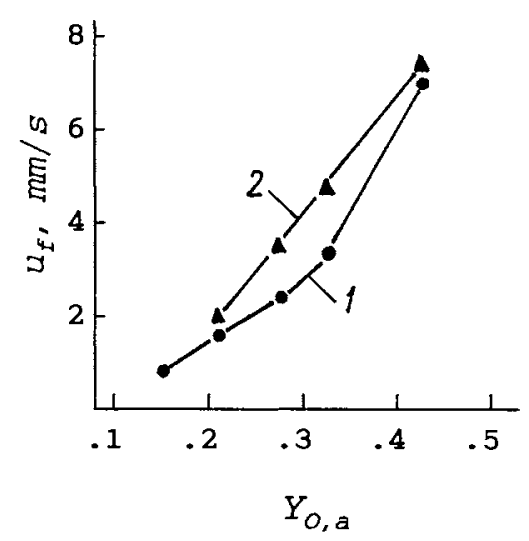

Fig.3 Dependence of flame spread rate upon the oxygen ambient mass fraction;

$L=0.1 \mathrm{~mm}$ : 1-present calculations, 2-measurements [28].

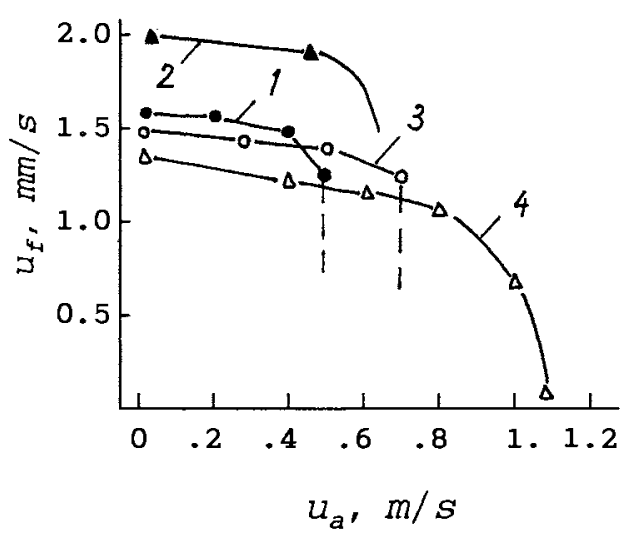

Fig.4 Dependence of flame spread rate upon the free-stream velocity; $L=0.1 \mathrm{~mm}$ : 1-present calculations, 2-measurements [28]. $L=0.13 \mathrm{~mm}$ : 3-present calculations, 4-measurements [26].

of free-stream velocity only. However, this feature does not relate to the proposed algorithm of flame spread rate prediction because the stationary solution is not achieved beyond the dashed lines of Fig.4 for any flame spread rate beginning from zero. The most probable reason of this limitation is caused by the very simplified model of gas flow dynamics since the momentum equations have been eliminated from the account.

The satisfactory agreement with well-known peculiarities of flame spread are obtained. Therefore, further investigations would be valuable. On the other hand, it is necessary to evaluate the validity of minimal entropy production principle specificly, i.e. by comparing the results obtained by this approach with another ones obtained by customary flame spread model which does not uses such physical assumption (as it was made for gas flame propagation [23]). In this regard a model like of Di Blasi et al. [13] which uses twodimensional unsteady equations seems to be the most reasonable object for comparison. The limitations and advantages would be performed by such comparison in the clearest form.

\section{ACKNOWLEDGEMENTS}

Authors express sincerest thanks to Professor T.Hirano for helpful discussion and providing the research funds for the first author who made a part of presented study being a visiting researcher at the University of Tokyo under the Postdoctoral Fellowship of Japan Society for the Promotion of Science. 


\section{REFERENCES}

1. Friedman, R., "A Survey of Knowledge about Idealized Fire Spread over Surfaces", Fire Research Abstracts and Review, 10:1-8, 1968.

2. Magee, R.S. and McAlevy, III, R.F., "The Mechanism of Flame Spread", J.Fire Flammabil., 2:271-297, 1971.

3. Williams, F.A., "Mechanisms of Fire Spread",Sixteenth Symposium (Int.) on Combustion, pp.1281-1294, The Combustion Institute, Pittsburgh, 1977.

4. Fernandez-Pello, A.C. and Hirano, T., "Controlling Mechanisms of Flame Spread", Combust. Sci.Technol., 32:1-31, 1983.

5. de Ris, J.N., "Spread of a Laminar Diffusion Flame",Twelfth Symposium (Int.) on Combustion, pp.241-252, The Combustion Institute, Pittsburgh, 1969.

6. Tarifa, C.S., Notario, P.P. and Torralbo A.M., "On the Process of Flame Spreading over the Surface of Plastic Fuel in an Oxidizing Atmosphere", Twelfth Symposium (Int.) on Combustion, pp.229-240, The Combustion Institute, Pittsburgh, 1969.

7. Sirignano, W.A., "A Critical Discussion of Theories of Flame Spread across Solid and Liquid Fuels", Combust. Sci. Technol, 6:95-105, 1972.

8. Sirignano, W.A., "Theory of Flame Spread above Solids",Acta Astronautica, 1:1285-1299, 1974.

9. Wichman, I.S. and Williams, F.A., "Comments on Rates of Creeping Spread of Flames over Thermally Thin Fuels", Combust. Sci. Technol., 33:207-214, 1983.

10. Delichatsios, M.A., "Exact Solution for the Rate of Creeping Flame Spread over Thermally Thin Materials", Combust. Sci. Technol., 44:257-267, 1986.

11. Fernandez-Pello, A.C. and Williams, F.A., "A Theory of Laminar Flame Spread over Flat Surfaces of Solid Combustibles", Combust. Flame, 28:251-277, 1977.

12. Frey, Jr., A.E., and Tien, J.S., "A Theory of Flame Spread over a Solid Fuel Including Finite-Rate Chemical Kinetics",Combust. Flame 36:263-289, 1979.

13. Di Blasi, C., Crescitelli S., Russo G. and Fernandez-Pello, A.C., "Model of the Flow Assisted Spread of Flames over a Thin Charring Combustible",Twenty-Second Symposium (Int.) on Combustion, pp.1205-1212, The Combustion Institute, Pittsburgh, 1988.

14. Bhattacharjee, S. and Altenkirch, R.A., "The Effect of Surface Radiation on Flame Spread in a Quiescent, Microgravity Environment", Combust.Flame, 84:160-169, 1991.

15. Wichman, I.S., "Theory of Opposed-Flow Flame Spread",Prog. Energ. Combust. Sci.,18:553-593, 1992.

16. Hirano, T., Sato, K., Sato, Y. and Sato, J., "Prediction of Metal Fire Spread in High Pressure Oxygen",_Combust. Sci. Technol., 32:137-159, 1983.

17. Sato, K. and Sega, S., "Smolder Spread in a Horizontal Layer of Cellulosic Powder", Second International Symposium on Fire Safety Sciences, pp.87-96, Hemisphere, New York, 1989.

18. Coveney, P.V., "The Second Law of Thermodynamics: Entropy, Irreversibility and Dynamics",_Nature, 333:409-415, 1988.

19. de Groot, S.R. and Mazur, P.,_Non-Equilibrium Thermodynamics, North Holland, Amsterdam, 1962.

20. Glansdorff, P. and Prigogine, I., Thermodynamic Theory of Structure, Stability and Eluctuations, Wiley, New York, 1971.

21. Gyarmati, I.,Non-Equilibrium Thermodynamics: Field Theory and Variational Principles, Springer-Verlag, Berlin, 1970.

22. Schechter, R.S., The Variational Method in Engineering, McGraw-Hill, New York, 1967. 
23. Karpov, A.I., "Minimal Entropy Production as an Approach to the Prediction of the Stationary Rate of Flame Propagation",J. Non-Equilib. Thermodyn., 17:1-9, 1992.

24. Hirano, T., Noreikis, S.E. and Waterman, T.E., "Postulations of Flame Spread Mechanisms", Combust. Flame, 22:353-363, 1974.

25. Frey, Jr., A.E. and Tien, J.S., "Near-Limit Flame Spread over Paper Samples", Combust. Elame, 26:257-267, 1976.

26. Hirano, T., Sato, K. and Tazawa, K., "Instability of Downward Flame Spread over Paper in an Air Stream", Combust. Flame, 26:191-200, 1976.

27. Altenkirch, R.A., Eichhorn, R. and Shang, P.C., "Buoyancy Effects on Flame Spreading Down Thermally Thin Fuels",Combust. Flame, 37:71-83, 1980.

28. Fernandez-Pello, A.C., Ray, S.R. and Glassman, I., "Flame Spread in an Opposed Forced Flow: The Effect of Ambient Oxygen Concentration",_Eighteenth Symposium (Int.) on Combustion, pp.579-589, The Combustion Institute, Pittsburgh, 1981.

29. Frey, Jr., A.E. and Tien, J.S., "A Theory of Flame Spread over a Solid Fuel Including Finite Rate Chemical Kinetics", Report No. FTAS/TR-77-134, Case Western Reserve University, Cleveland, 1977.

30. Patankar, S.V., Numerical Heat Transfer and Fluid Elow, Hemisphere, New York, 1980. 31. de Donder, T. and van Rysselberghe, P., Thermodynamic Theory of Affinity, Stanford University Press, Stanford, 1936.

\section{APPENDIX}

According to the mathematical model of solid fuel described by Eq.(30) where diffusion and viscous motion are not considered, the entropy is produced by heat transfer and chemical reaction. Generalized forces due to these processes are [19]:

$$
X_{T}=-\frac{1}{T_{s}^{2}} \frac{d T_{s}}{d x}
$$

$$
X_{W}=A_{s} / T_{s}
$$

The thermodynamic fluxes described by Fourier's law for thermal conduction and by chemical reaction's rate are following:

$$
J_{T}=-\lambda_{s} d T_{s} / d x
$$

$$
J_{W}=\rho_{s} W_{s}
$$

Pyrolysis reaction has an endothermic effect and it can exist only due to the external heat flux from the gas phase. Therefore, thermal destruction of material occurs far from the equilibrium state (where chemical affinity tends to zero) and pyrolysis reaction can be considered as an initial stage of global chemical process which includes gas-phase combustion as well. In this case pyrolysis reaction's chemical affinity $\boldsymbol{A}_{s}$ can be assumed [31] equal to the heat effect $Q_{s}$. Using Eqs.(A-1)-(A-4) the entropy production in the solid fuel is expressed by Eq.(31). 